\title{
ELASTIC PROPERTIES OF MULTI-COMPONENT NICKEL SOLID SOLUTIONS
}

\author{
Kuiying Chen ${ }^{1}$, Linruo Zhao ${ }^{1}$, Prakash C. Patnaik ${ }^{1}$, John S. Tse ${ }^{2}$ \\ ${ }^{1}$ Structures, Materials and Propulsion Laboratory, Institute for Aerospace Research, National Research Council Canada, \\ Ottawa, Ontario, K1A 0R6, Canada \\ ${ }^{2}$ Steacie Institute for Molecular Sciences, National Research Council Canada, Ottawa, Ontario, K1A 0R6, Canada
}

Keywords: Elastic property, Ni solid solution, ab initio calculation

\begin{abstract}
The effects of alloying additions on elastic properties of multicomponent $\mathrm{Ni}$ solid solutions have been systematically investigated using $a b$ initio density functional theory calculations within the generalized gradient approximation (GGA). An elastic property map involving 60 purposely-chosen compositions including binary, ternary, quaternary and high-order (up to 6) $\mathrm{Ni}$ solid solutions has been created. Analyses of the map revealed that alloying additions could enhance elastic moduli up to the quaternary system, beyond which further alloying would slightly weaken elastic moduli. The enhancement of elastic moduli by alloying additions is more evident for lower order systems than for higher order systems. Based on the calculated elastic moduli, the ductile/brittle behavior of the materials has been briefly assessed. The current research has, for the first time, generated a calculated elastic modulus database for $\mathrm{Ni}$ solid solutions that could be used for future design of new superalloys.
\end{abstract}

\section{Introduction}

Ni-base single crystal (SC) superalloys, which are used for turbine blades and vanes in advanced aero engines have a characteristic microstructure with cuboidal $\gamma^{\prime}$ precipitates coherently embedded in the Ni solid solution $(\gamma)$ matrix [1]. In order to improve hightemperature creep-rupture strength and fatigue life of superalloys, selected refractory elements are added to achieve solid solution strengthening, primarily through the strengthening of the $\gamma$ matrix [2]. From the engine design standpoint, elastic properties are also important because they have a major influence on the thermal and mechanical behavior of rotating components [3]. Alloying elements that are typically added into superalloys strongly affect elastic properties of Ni $\gamma$ matrix. Assessing the underlying effects in a systematic manner based on the period table is an essential step towards the design of new alloy systems. This paper reports the first systematic $a b$ initio density functional theory [4] calculations of elastic properties of Ni solid solutions with singleand multi-component alloying additions. The alloying elements are $3 d, 4 d$ and $5 d$ B-group transition metals in the periodical table, including refractory-, platinum-, noble and other metals. An elastic property-composition map for binary, ternary, quaternary and high order systems has been created, which contains useful information on alloying strengthening effects.

\section{Calculation Methodology}

The Vienna ab initio simulation package (VASP) [5,6,7], which is based on density functional theory within the generalized gradient approximation (GGA) [8], was utilized to calculate the elastic constants and moduli of multi-component $\mathrm{Ni}$ solid solutions. The electron-ion interaction is described by an ultrasoft pseudopotentials proposed by Vanderbilt [9]. As elastic constants are derived from the second derivative of calculated total energy, all the essential numerical parameters in the calculations were checked carefully to ensure the precision of the results. In order to achieve a high level of convergence of the total energy, a highenergy cut-off was chosen. The planewave cutoff energy is 290 $\mathrm{eV}$ and the convergence of the calculations is within $1 \sim 2 \mathrm{meV}$. The integration in the Brillion zone was performed by using Monkhorst Pack $k$-points. The elastic constants were found to be almost the same if the $k$-point sampling was with sets of $6 \times 6 \times 6$ and $8 \times 8 \times 8$, in which the latter $k$-point sampling was used in the calculation. The maximum strain used in the total energy fitting to derive elastic moduli is within $1 \%$.

In principle, according to the definition of a supercell (repeated periodically along 3-dimentions), the calculated results are independent on the selection of cell model size. The lattice constants for $\mathrm{Ni}$ solid solutions with alloying additions are determined after the relax of a supercell. In order to investigate the multi-component alloying effect on the elastic properties of $\mathrm{Ni}$ solid solutions, a large supercell model has been constructed as shown in Figure 1(a). To present the mode clearly, the supercell was divided into 8 identical blocks, with each block as a facecentered-cubic (FCC) closed pack sub-unit, as shown in Figure 1(b).

(a)

(b)
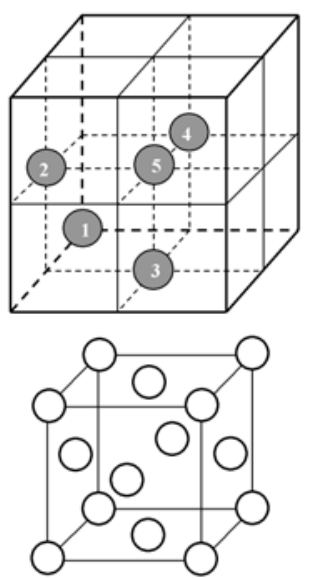

Figure 1. (a) A large supercell model is divided into 8 identical blocks. (b) Each block as a sub-unit cell shows a face-centredcubic (FCC) pack structure. The numbered grep circles represent alloying atoms, while open circles represent $\mathrm{Ni}$ atoms.

According to the symmetry of the model, the supercell consists of total 32 inequivalent atoms, which are grouped into the numbered 
grey circles as substitution alloying atoms and open circles as $\mathrm{Ni}$ atoms. In order to minimize the interactions between the alloying atoms, the substitutional elements were separated beyond the first nearest neighbor distance. Such an arrangement renders the solid solutions as approximate dilute systems for the binary and ternary systems. The maximum alloying concentration in the model was approximately around 15 at \%, with up to five different alloying elements. For Ni solid solutions with a cubic symmetry, there are three independent elastic constants, i.e., $\mathrm{C}_{11}, \mathrm{C}_{12}$ and $\mathrm{C}_{44}$ [10], which can be determined by introducing tetragonal and trigonal lattice distortions (see the previous publications $[11,12]$ ). The bulk modulus $B$ can be directly derived from the second derivative of calculated total energy $E_{t o t}$ as a function of isotropic volume variation. Using $\mathrm{C}_{11}, \mathrm{C}_{12}$ and $\mathrm{C}_{44}$, the shear modulus $G$, Young's modulus $E$, and the Poisson's ratio $v$ can be calculated using the Voigt-Reuss-Hill bound formula [13].

\section{Results And Discussions}

\section{Alloying Effect On Elastic Properties}

For binary Ni-X solid solutions, two alloying atoms that occupy the numbered 1 and 5 grey circle positions in Figure 1(a) were added to substitute for $\mathrm{Ni}$ atoms. This gave an alloying concentration around 6 at $\%$. The separation between the two alloying atoms was beyond the second nearest neighbor distance to minimize their interactions. Using this model, the effect of
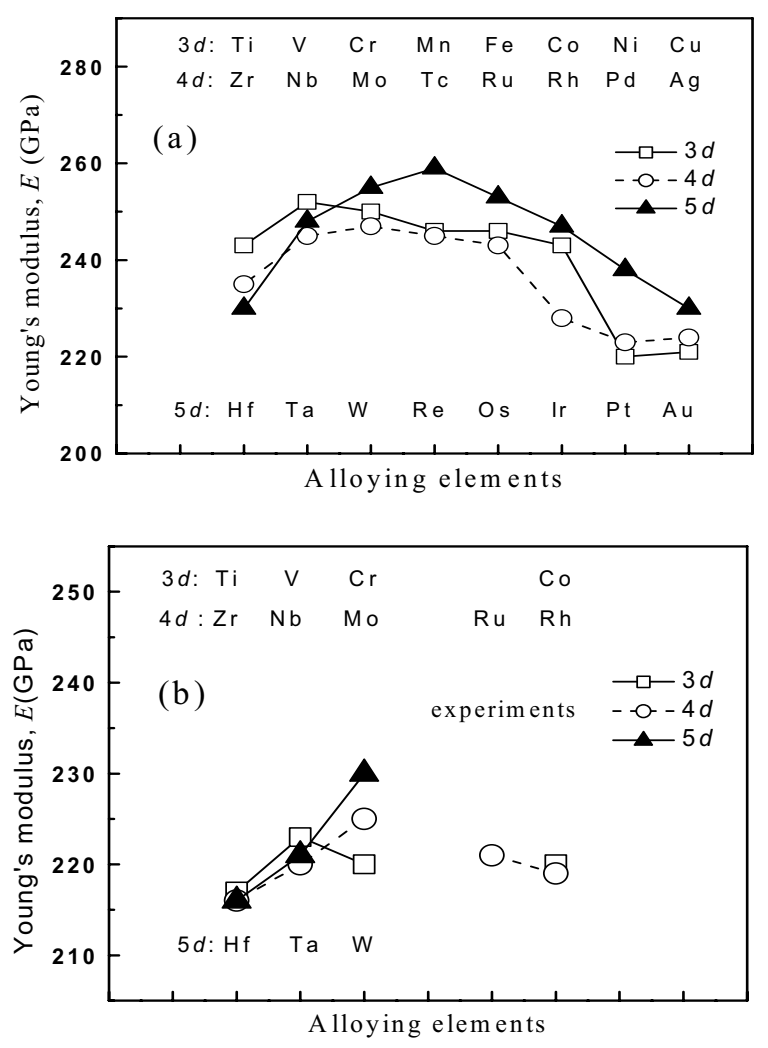

Figure 2. (a) The calculated Young's modulus $E$ as a function of alloying additions for $3 d, 4 d$ and $5 d$ metals in the periodical table. (b) Some experimentally measured Young's modulus [14]. individual alloying elements $\mathrm{X}$ on elastic properties of the binary solutions was studied. Figure 2(a) presents the calculated Young's modulus $(E)$ as a function of the alloying additions from the period table for $3 d, 4 d$ and $5 d$ metals. It is interesting to note that all the alloying additions give rise to a common trend with Young's modulus from each of the $3 d, 4 d$ and $5 d$ metals yielding a maximum value at a certain B-group element. For $3 d$ transition metals, the maximum $E$ is obtained by $\mathrm{V}$ alloying, while for $4 d$ and $5 d$ metals, the maximum $E$ values are generated from Mo and Re alloying, respectively. Figure 2(b) shows some Young's modulus values obtained from experimental measurements [14]. Comparing Figure 2(a) with Figure 2(b), a similar trend between our calculations and previous experiments can be observed. The calculated $E$ values are about $10 \%$ larger than those from experiments, probably due to the fact that the experimental materials are polycrystalline and contain defects, while our calculation models represent defect-free single crystals.

It is known that the trend of certain mechanical properties could be estimated to some extent through the analysis of elastic moduli. Pugh [15] proposed that the resistance of a material to plastic deformation is related to the product $G \mathbf{b}$, where $G$ and $\mathbf{b}$ are the shear modulus and Burgers vector, respectively. Furthermore, the fracture strength of a material was assumed to be proportional to the product $B a$, where $B$ and $a$ are bulk modulus and lattice parameter, respectively. The ratio $G / B$ then reflects the competition between the shear and cohesive strengths at a crack tip. If $G / B<0.4$, the material behaves in a ductile manner; if $G / B$ $>0.5$, the material behaves in a brittle manner. For instance, as a typical ductile material $\mathrm{Al}$ has a $G / B$ value of 0.37 , while as a brittle semiconductor $\mathrm{Si}$ has a $G / B$ value of 0.70 [16]. This criterion has been recently applied in the study of brittle vs. ductile transition for intermetallic compounds from first principles calculations [16,17]. From Table I, alloying element $\mathrm{V}$ yields a better ductile effect by lowering $G / B$ value $(0.379)$ compared to other elements. For $5 d$ metals, refractory $\mathrm{W}$ achieves a similar ductile effect as $\mathrm{V}$, but $\mathrm{Re}$ shows a slightly brittle effect by increasing $G / B(0.478)$.

Elastic properties of multi-component Ni solid solutions have also been calculated and analyzed. The results are presented in Tables II, III and IV for the ternary, quaternary and high order systems, respectively. The alloying elements for multi-component solid solutions are purposely chosen, with an aim to identify the underlying roles of these elements in affecting elastic properties of superalloys. In particular, the strengthening effect of refractory and platinum-group metals, such as $\mathrm{V}, \mathrm{Mo}, \mathrm{W}$, and Re were investigated. The compositions were chosen as follows: one major alloying concentration was kept as a constant, while others (up to 4 elements) were varied step by step. For instance, in Ni-3 at. $\% \mathrm{~W}-X_{2}$ at. $\% \operatorname{Re}-X_{3}$ at. $\% \mathrm{Ru}-X_{4}$ at. $\% \mathrm{Mo}-X_{5}$ at. $\% \mathrm{Cr}$, the concentration of $\mathrm{W}$ was kept as 3 at. \%, while the concentrations of other alloying elements $X_{i}$ were varied systematically in the range between 0 and 15 at. $\%, i=2$ to 5 . In this way, an elastic modulus-composition map has been constructed in the form of several property-composition tables.

Table II shows the calculated elastic constants and moduli for the ternary system. The base alloy Ni-6Re is chosen from Table I. The ternary alloy system is $\mathrm{Ni}-3 \mathrm{Re}-3 \mathrm{X}$, i.e., one $\mathrm{Re}$ atom was replaced by an alloying atom $X$. It is noteworthy in Table II that alloying additions in the ternary alloys have little effect in further enhancing elastic constants and moduli. This phenomenon can be 
Table I. Alloying effect on elastic constants $\left(\mathrm{C}_{11}, \mathrm{C}_{12}\right.$ and $\left.\mathrm{C}_{44}\right)$, moduli (bulk modulus $B$, shear modulus $G$ and Young's modulus $E$ ) and Poisson ratio $v$ of binary Ni solid solutions. The unit for elastic modulus is GPa.

\begin{tabular}{|c|c|c|c|c|c|c|c|c|c|}
\hline \multicolumn{2}{|c|}{ Binary Ni-X } & $B$ & $G$ & $E$ & $G / B$ & $\mathrm{C}_{11}$ & $\mathrm{C}_{12}$ & $\mathrm{C}_{44}$ & $v$ \\
\hline $\mathrm{Ti}$ & \multirow{7}{*}{$3 d$} & 194 & 93 & 242 & 0.478 & 282 & 150 & 119 & 0.292 \\
\hline $\mathrm{V}$ & & 252 & 96 & 253 & 0.379 & 325 & 216 & 137 & 0.332 \\
\hline $\mathrm{Cr}$ & & 197 & 97 & 250 & 0.489 & 282 & 155 & 129 & 0.289 \\
\hline Mn & & 185 & 96 & 248 & 0.52 & 267 & 144 & 132 & 0.276 \\
\hline $\mathrm{Fe}$ & & 199 & 95 & 247 & 0.48 & 277 & 159 & 131 & 0.293 \\
\hline $\mathrm{Co}$ & & 194 & 95 & 245 & 0.488 & 276 & 156 & 131 & 0.29 \\
\hline $\mathrm{Cu}$ & & 220 & 84 & 224 & 0.384 & 280 & 189 & 128 & 0.33 \\
\hline $\mathrm{Zr}$ & \multirow{7}{*}{$4 d$} & 237 & 88 & 235 & 0.372 & 312 & 199 & 119 & 0.331 \\
\hline $\mathrm{Nb}$ & & 220 & 94 & 246 & 0.426 & 291 & 184 & 136 & 0.314 \\
\hline Mo & & 220 & 95 & 248 & 0.431 & 293 & 183 & 139 & 0.312 \\
\hline $\mathrm{Ru}$ & & 212 & 93 & 244 & 0.427 & 290 & 182 & 135 & 0.313 \\
\hline $\mathrm{Rh}$ & & 193 & 87 & 230 & 0.46 & 262 & 157 & 127 & 0.301 \\
\hline $\mathrm{Pd}$ & & 192 & 87 & 225 & 0.451 & 252 & 154 & 126 & 0.306 \\
\hline $\mathrm{Ag}$ & & 225 & 85 & 228 & 0.38 & 287 & 191 & 128 & 0.331 \\
\hline $\mathrm{Hf}$ & \multirow{7}{*}{$5 d$} & 207 & 88 & 230 & 0.424 & 281 & 169 & 118 & 0.315 \\
\hline $\mathrm{Ta}$ & & 245 & 93 & 248 & 0.381 & 315 & 208 & 135 & 0.331 \\
\hline W & & 228 & 97 & 254 & 0.424 & 303 & 190 & 140 & 0.315 \\
\hline $\mathrm{Re}$ & & 210 & 100 & 259 & 0.478 & 273 & 168 & 168 & 0.294 \\
\hline Ir & & 225 & 91 & 240 & 0.5 & 295 & 190 & 131 & 0.322 \\
\hline $\mathrm{Pt}$ & & 225 & 91 & 240 & 0.403 & 295 & 190 & 131 & 0.322 \\
\hline $\mathrm{Au}$ & & 212 & 88 & 232 & 0.413 & 287 & 196 & 120 & 0.318 \\
\hline
\end{tabular}

understood from the relationship between elastic moduli and the interatomic bonding strength [18], i.e., the bonding strength of $\mathrm{Ni}$ $\mathrm{Re}$ atomic pair could be stronger than that between $\mathrm{Ni}$ and other alloying elements $\mathrm{X}$. This relationship is evident for $\mathrm{V}, \mathrm{W}$ and Mo additions in Ni-X binary solid solutions in Table I.

Table III shows the calculated elastic moduli for the quaternary system. As for the ternary system, the alloys are in the form of $\mathrm{Ni}$ $3 \mathrm{Re}-3 \mathrm{Mo}-3 \mathrm{X}$. The maximum alloying concentration is around 10 at $\%$. Compared to the binary and ternary systems, the shear and Young's moduli of the quaternary system are increased by about $9 \sim 15 \%$ in general. Table IV shows the calculated elastic constants and moduli for high order (5 and 6) Ni solid solutions. It was observed that high order alloying additions could slightly weaken elastic moduli compared to the quaternary system. This effect could arise from complex interactions between alloying elements in high order systems. These interactions depend on the detailed arrangements of alloying atoms presented in the grey circle positions in Figure 1(a). As a result, the alloying effect on elastic moduli for individual additions observed in binary and ternary systems does not simply apply in the high order systems. It was found that for binary, ternary, quaternary and high-order (up to 5) Ni solid solutions, as the separations between these substitution solute atoms are beyond the first nearest neighbor distances shown in Figure 1(a) by numbered grey circles 1, 2, 3 and 4 , the calculated total energies of supercell systems are independent on the choice of locations for substitution atoms.
Thus the elastic constants and moduli derived from the second derivative of the calculated energy are independent on the selection of location for a substitution element. In the case of 6element $\mathrm{Ni}$ solute solutions, as the separations between the numbered 5 substitution atom and other solute atoms (numbered $1,2,3$ and 4) are in the first neighbor range, the interaction between these solute atoms may have some effects on the calculated total energy of a supercell. The calculated elastic constants due to the exchange of location for substitution elements indicate that these effects are within $6.0 \%$. A more systematic approach to this problem will require a combination of $a b$ initio density functional theory and cluster variational methods (CVM) calculations, in which the latter method consider the entire arrangements of alloying atoms based on the energy minimization.

\section{Alloying Effect on Solid Solution Strengthening}

It is widely accepted that solid solution strengthening of alloys may involve several mechanisms [19,20]. For elastic interactions between the strain fields of a solute and dislocations, Fleishcher [18] suggested that the degree of solid solution strengthening could be correlated with an interaction parameter defined as the combined effects of atomic size and shear modulus. However, Mishima et al [21] has demonstrated that the atomic size misfit 
Table II. Alloying effect on elastic constants, modulus and Poisson ratio of ternary Ni-Re-X solid solutions The unit for moduli is GPa.

\begin{tabular}{|c|c|c|c|c|c|c|c|c|c|}
\hline \multicolumn{2}{|c|}{ Ternary Ni-Re-X } & $B$ & $G$ & $E$ & $G / B$ & $\mathrm{C}_{11}$ & $\mathrm{C}_{12}$ & $\mathrm{C}_{44}$ & $v$ \\
\hline $\mathrm{Ti}$ & \multirow{4}{*}{$3 d$} & 225 & 93 & 246 & 0.414 & 297 & 188 & 133 & 0.318 \\
\hline $\mathrm{V}$ & & 245 & 96 & 255 & 0.39 & 321 & 209 & 138 & 0.327 \\
\hline $\mathrm{Cr}$ & & 223 & 94 & 248 & 0.422 & 300 & 185 & 131 & 0.315 \\
\hline $\mathrm{Co}$ & & 237 & 91 & 243 & 0.385 & 308 & 201 & 131 & 0.329 \\
\hline $\mathrm{Y}$ & \multirow{6}{*}{$4 d$} & 223 & 85 & 225 & 0.379 & 289 & 190 & 121 & 0.332 \\
\hline $\mathrm{Zr}$ & & 232 & 88 & 237 & 0.381 & 301 & 197 & 127 & 0.33 \\
\hline $\mathrm{Nb}$ & & 240 & 93 & 248 & 0.389 & 312 & 204 & 134 & 0.328 \\
\hline Mo & & 241 & 94 & 250 & 0.391 & 313 & 203 & 136 & 0.327 \\
\hline $\mathrm{Ru}$ & & 232 & 92 & 246 & 0.398 & 304 & 196 & 133 & 0.323 \\
\hline $\mathrm{Rh}$ & & 221 & 86 & 229 & 0.39 & 288 & 188 & 124 & 0.328 \\
\hline $\mathrm{Hf}$ & \multirow{4}{*}{$5 d$} & 227 & 87 & 231 & 0.383 & 294 & 193 & 125 & 0.33 \\
\hline $\mathrm{Ta}$ & & 243 & 94 & 252 & 0.389 & 316 & 207 & 136 & 0.328 \\
\hline $\mathrm{W}$ & & 244 & 96 & 255 & 0.394 & 318 & 206 & 138 & 0.326 \\
\hline Ir & & 232 & 93 & 245 & 0.4 & 305 & 196 & 132 & 0.324 \\
\hline
\end{tabular}

Table III. Alloying effect on elastic constants, moduli (GPa) and Poisson ratio of quaternary Ni-ReMo-X solid solutions

\begin{tabular}{|c|c|c|c|c|c|c|c|c|c|}
\hline \multicolumn{2}{|c|}{ Quaternary } & $B$ & $G$ & $E$ & $G / B$ & $\mathrm{C}_{11}$ & $\mathrm{C}_{12}$ & $\mathrm{C}_{44}$ & $v$ \\
\hline $\mathrm{Ti}$ & & 235 & 111 & 287 & 0.471 & 295 & 205 & 199 & 0.296 \\
\hline $\mathrm{V}$ & $3 d$ & 234 & 110 & 286 & 0.472 & 294 & 204 & 198 & 0.296 \\
\hline $\mathrm{Cr}$ & & 235 & 95 & 251 & 0.405 & 314 & 196 & 131 & 0.321 \\
\hline $\mathrm{Zr}$ & & 241 & 126 & 322 & 0.523 & 330 & 196 & 192 & 0.277 \\
\hline $\mathrm{Nb}$ & $4 d$ & 245 & 114 & 297 & 0.467 & 306 & 214 & 169 & 0.298 \\
\hline $\mathrm{Ru}$ & & 251 & 100 & 265 & 0.398 & 311 & 221 & 169 & 0.324 \\
\hline Hf & & 237 & 102 & 267 & 0.429 & 287 & 212 & 193 & 0.312 \\
\hline $\mathrm{Ta}$ & & 248 & 117 & 302 & 0.47 & 310 & 217 & 212 & 0.297 \\
\hline W & $5 d$ & 223 & 85 & 225 & 0.379 & 289 & 190 & 121 & 0.33 \\
\hline $\mathrm{Ir}$ & & 246 & 101 & 267 & 0.412 & 307 & 216 & 172 & 0.319 \\
\hline
\end{tabular}

and modulus misfit cannot completely explain the solid solution strengthening effect induced by transition metal elements. Thus, they proposed an electron valence mechanism to account for the extra strengthening effect. Later, Cottrell et al [22] introduced the mechanism related to electric interactions from the static electric filed of free electrons, while Morinaga et al suggested $[23,24]$ that the extra strengthening effect might come from the strong $d$-electron hybridization between solute and solvent atoms.

As a macroscopic measure of materials stiffness, elastic constants and moduli could provide useful information on interatomic forces and dislocation behavior related to Peierls-Nabarro stress.
Clerc et al [18] proposed that the bulk modulus $B$ could be used as a measure of average atomic bond strength because it has a strong correlation with the cohesive energy or binding energy of atoms in crystal. Assuming this correlation is also true for Young's modulus, it can be observed from Figure 2(a) and Table I that $\mathrm{V}$ has the maximum bonding strengthening effect among $3 d$ transition metals, while Mo and Re have the maximum bonding strengthening effect among $4 d$ and $5 d$ metals, respectively.

As shown in Tables II, III and IV, the quaternary alloying additions can achieve the best bonding strengthening effect in $\mathrm{Ni}$ solid solutions among the binary, ternary and quaternary systems. This is also true by comparing the quaternary system with the 
Table IV. Alloying effect on elatsic constants, moduli (GPA) and Poisson ratio of high order Ni solid solutions.

\begin{tabular}{|c|c|c|c|c|c|c|c|c|c|}
\hline \multicolumn{2}{|c|}{ Ni-ReMoCr-X } & $B$ & $G$ & $E$ & $G / B$ & $\mathrm{C}_{11}$ & $\mathrm{C}_{12}$ & $\mathrm{C}_{44}$ & V \\
\hline $\mathrm{Ti}$ & \multirow{2}{*}{$3 d$} & 234 & 93 & 246 & 0.396 & 306 & 198 & 133 & 0.325 \\
\hline V & & 233 & 91 & 241 & 0.39 & 302 & 198 & 132 & 0.327 \\
\hline $\mathrm{Zr}$ & \multirow{3}{*}{$4 d$} & 238 & 87 & 232 & 0.365 & 313 & 200 & 1160 & 0.332 \\
\hline $\mathrm{Nb}$ & & 245 & 93 & 248 & 0.38 & 316 & 209 & 135 & 0.331 \\
\hline $\mathrm{Ru}$ & & 241 & 101 & 267 & 0.2 & 324 & 199 & 140 & 0.316 \\
\hline Hf & \multirow{3}{*}{$5 d$} & 236 & 88 & 234 & 0.371 & 312 & 198 & 117 & 0.335 \\
\hline $\mathrm{Ta}$ & & 246 & 94 & 251 & 0.383 & 318 & 210 & 137 & 0.33 \\
\hline $\mathrm{Ir}$ & & 245 & 103 & 271 & 0.421 & 330 & 202 & 142 & 0.315 \\
\hline \multicolumn{10}{|c|}{ Ni-ReMoCrHf-X } \\
\hline $\mathrm{Ti}$ & \multirow{2}{*}{$3 d$} & 245 & 86 & 232 & 0.353 & 318 & 208 & 117 & 0.342 \\
\hline $\mathrm{V}$ & & 244 & 100 & 263 & 0.409 & 350 & 191 & 116 & 0.32 \\
\hline $\mathrm{Nb}$ & \multirow{3}{*}{$4 d$} & 256 & 91 & 245 & 0.327 & 338 & 215 & 119 & 0.334 \\
\hline $\mathrm{Ru}$ & & 252 & 92 & 247 & 0.367 & 339 & 209 & 117 & 0.337 \\
\hline $\mathrm{Zr}$ & & 255 & 89 & 239 & 0.349 & 332 & 217 & 119 & 0.336 \\
\hline $\mathrm{Ta}$ & \multirow{2}{*}{$5 d$} & 257 & 91 & 245 & 0.356 & 338 & 216 & 120 & 0.338 \\
\hline Ir & & 256 & 95 & 252 & 0.3691 & 345 & 211 & 119 & 0.332 \\
\hline
\end{tabular}

high order systems (up to 6). Therefore, alloying additions beyond four elements may not necessarily make more contributions to the enhancement of elastic moduli for Ni solid solutions. However, more alloying additions, particularly refractory alloying additions, could lead to stronger elastic interactions between the strain fields of solutes and dislocations, which results in larger solid solution strengthening effects from plastic yielding point of view [2]. Nevertheless, the new knowledge generated from this research could be useful for future design of advanced superalloys.

\section{Conclusions}

The effects of alloying additions on elastic properties of 60 purposely-chosen multi-compositions Ni solid solutions have been systematically investigated using $a b$ initio density functional theory calculations. The calculated Young's modulus for binary $\mathrm{Ni}$ solid solutions containing $3 d, 4 d$ and $5 d$ alloying elements from the periodical table present a similar trend as those from experimental observations. The quaternary alloying system shows the best strengthening effect in terms of elastic properties by comparison with the binary, ternary and high order systems. From plastic yielding standpoint, other strengthening mechanisms such as solute-dislocation interactions could play a more important role for alloying additions beyond the quaternary system

\section{Acknowledgement}

The authors wish to acknowledge the financial support from the Department of National Defence Canada through the Technology Investment Fund Program.

\section{References}

1. C. T. Sims, Superalloys II (John Wiley \& Sons, New York, NY, 1987), 97-133.

2. M. Durand-Charre, The microstructure of superalloys (Gordon and Breach Science Publishers, 1999), 1-14.

3. B.P. Bewlay et al., "A Review of Very-High-Temperature NbSilicide-Based Composites”, Metall. Mater. Trsns., A34, (2003), 243-2052.

4. D. A. Dixon, J. Andzelm, G. Fitzerald, E. Wimmer and P. Jasien, Density Functional Methods in Chemistry, ed. J. K. Labanowski, and J. W. Andzelm (Berlin: Springer, 1991), 33-56.

5. G. Kresse and J. Furthmuller, "Efficiency of ab-initio Total Energy Calculations for Metals and Semiconductors Using a Plane-Wave Basis Set", Comput. Math. Sci. ,6,(1996), 15-50.

6. G. Kresse and J. Furthmuller, "Efficiency Iterative Schems for ab-initio Total Energy Calculations Using a Plane-Wave Basis Set", Phys. Rev. B 54, (1996), 11169-11186.

7. G. Kresse and J. Hafner, " $a b$ initio Simulation of the Metal/Nometal Transition in Expanded Fluid Mercury", Phys.Rev. B 55, (1997), 7539-7548.

8. J. P. Perdew and Y. Wang, "Accurate and Simple Analytic Representation of the Electron-Gas Correlation Energy", Phys. Rev. B 46, (1992), 13244-13249. 
9. D. Vanderbilt, "Soft Self-Consistent Pseudopotentials in a Generalized Eigenvalue Formalism”, Phys. Rev. B 41, (1990), 7892-7895.

10. J. Chen et al., "Elastic Constants of $\mathrm{NbC}$ and $\mathrm{MoN}$ : Instability

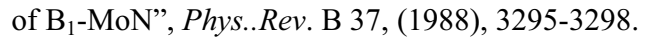

11. K. Chen, L.R. Zhao, and J. Tse, "ab initio Study of Elastic Properties of Ir and $\mathrm{Ir}_{3} \mathrm{X}$ compounds", J. Appl. Phys., 93,(2003), 2414-2417.

12. K. Chen, L.R. Zhao, and J. Tse, "Alloying Effects on Elastic Properties of TiN-Based Nitrides", J. Phys. D:Appl. Phys.,36, (2003), 2725-2729.

13. M. Nakamura, "Elastic Properties", Intermetallics Compounds, Vol.1, Principles, ed. J. H. Westbrook and R. L. Fleischer (1994, John Wiley and Sons Ltd), 873-893.

14. Y. Mishima et al, "Solid Solution Hardening of Ni- Role of Transition Metal and B-Subgroup Solutes", Trans. Jap. Inst. Met., 27, (1986), 656-664.

15. S. F. Pugh, "Relation Between the Ealstic Moduli and the Plastic Properties of Polycrystalline Pure Metals", Phil. Mag., 45, (1954), 823-842.

16. D. G. Pettifor, "Prediction of Structure and Properties of Intermetallics", Mater. Sci. Techn., 8, (1992)345-349.

17. A.T. Paxton and D. G. Pettifor, "Al3Ru-A Ductile Trialuminide", Script. Metall., 26, (1992), 529-533,

18. D. G. Clerc and H. M. Ledbetter, "Mechanical Hardness: A Semiempirical Theory Based on Screened Electrostatics and Elastic Shear", J. Phys. Chem. Solids, 59, 1071(1998), 1071-1095.

19. R. L. Fleishcher, "Substitutional Solution Hardening", Act. Met., 8, (1963), 203-209.

20. R. L. Fleishcher, and E. Hornbogen, "Theory of Working Hardening”, Phys.Stat.Sol., 12, (1965), 235-250.

21.Y. Mishima et al., "Solid Sulution Hardening of Ni3Al with Ternary Additions:, Trans. Japan. Institu. Metals., 27, (1986), 648-655.

22. A. H. Cottrell, S.C. Hunter and F. R. N. Nabarro, Phil. Mag. 44, (1953), 1064.

23. M. Morinaga, N. Yukawa and H. Adachi, "Alloying Effect on the Electronic Structure of $\mathrm{Ni}_{3} \mathrm{Al}\left(\gamma^{\prime}\right)$ ", J. Phys. Soc. Japan, 53, (1984), 653-663.

24. M. Morinaga et al., "New PHACOMP and its Applications to Alloys to Alloy Design", Superalloys (TMS, Warrendale, PA, U.S.A, 1984), 523-532. 